\title{
Isolation and expression profiles of class III $P R X$ gene family under drought stress in Camellia sinensis
}

\author{
H.J. LI, H.B. WANG, Y. CHEN, Q.P. MA, Z. ZHAO, X.H. LI, and X. CHEN* \\ Tea Research Institute, Nanjing Agricultural University, Weigang Road 1, Nanjing 210095, \\ Jiangsu, P.R. China
}

\begin{abstract}
The class III PRX family is a class of heme-containing oxidases and plays important roles in response to abiotic stress in plants. The responses to abiotic stresses could be regulated by phytohormones like abscisic acid (ABA) and methyl jasmonate (MeJA). In this research, 11 CsPRXs genes in tea plant (Camellia sinensis) were cloned and analyzed. Based on the similarity of the sequences, they were classified into 5 sub-groups. According to the results of reverse transcription PCR, CSPRX55 presented the highest expression in roots compared to stems and leaves of both tea cultivars LJ43 and Baiye 1. Besides, the expressions of CsPRX12 and CsPRX73 were highest in roots, while CsPRX4, CsPRX47, and CsPRX72 were highest in stems of 'Baiye 1'. But most of CsPRXs showed the highest expressions in leaves of 'LJ43'. CsPRXs appeared different expression patterns under drought stress of tea plants which were pre-treated with ABA or MeJA for three days. In 'LJ43' and 'Baiye 1', there were $3 C s P R X s$ and $7 C s P R X s$ up-regulated by exogenous ABA and MeJA, respectively. However, CsPRX4 and CsPRX16 were down-regulated in 'LJ43' treated with ABA and MeJA. It suggested that CsPRXs possessed diverse functions in response to hormones and abiotic stress. In 'LJ43', the activity of peroxidase (POD) was increased when pre-treated by ABA and MeJA, and the highest activity appeared after 24 and $12 \mathrm{~h}$, respectively. In 'Baiye 1', the activity of POD was also increased, however, when pre-treated by MeJA, the peak-time of POD activity was at $24 \mathrm{~h}$. But the change had no obvious rule after ABA treatment. Exogenous ABA or MeJA may play a role in protecting tea plants suffered from drought stress via regulating some $C s P R X s$ expressions and increasing POD activity.
\end{abstract}

Additional key words: abscisic acid, gene expression, methyl jasmonate, peroxidase activity, tea plant.

\section{Introduction}

Tea plant is an important economic crop especiallly in China, India, Sri Lanka, and Kenya. In summer, some plants are suffered from drought-resulting osmotic stress (Bartels and Sunkar 2005) which would lead to a decline on tea yield and quality.

The responses of plants to favourable or unfavourable environments are based on multi-gene families. Their functional diversity was obtained by mutation and reorganization from an ancestral genes (Wu et al. 2009). The class III PRX family is a class of heme-containing oxidases found in microorganisms, plants, and animals. In plants, they played vital roles in dealing with various stresses, in synthesis of lignin and other cell wall components, in metabolism of auxin, and in elimination of toxins and indicators of aging (Kunieda et al. 2013, Wang et al. 2015).
Class III peroxidase (class III PRXS) is usually used according to the gene annotation, but the abbreviation for related enzyme is peroxidase (POD, EC 1.11.1.7). Peroxidases remove reactive oxygen in plants and they are also considered as a potentially important components of plant signal transduction pathways (McInnis et al. 2005). Bioinformatics of the PRXS family had been studied in Arabidopsis thaliana (Michael et al. 2002, Cosio and Dunand 2008), Oryza sativa (Passardi et al. 2004), Populus euphratica (Ren et al. 2014), Zea mays (Wang et al. 2015), and Pyrus bretschneideri (Cao et al. 2016). Besides, POD activity is always measured as an indicator when plants were suffered by stresses (Wang and Chen 2011, Mirzaee et al. 2013). In the condition of drought stress, the expression of $P R X$ genes are often increased. In the wheat, the expression of $P R X 1$ increases significantly after drought treatment (Sharma et al. 2013). Also, the number of POD isoforms can be affected by drought

Submitted 26 February 2019, last revision 7 September 2019, accepted 19 September 2019.

Abbreviations: ABA - abscisic acid, MeJa - methyl jasmonate, POD - peroxidase.

Acknowledgment: This work was supported by the Modern Agro-industry Technology Research System (CARS-19), the National Science Foundation of China (31470690), and the Modern Agriculture Industry System in Jiangsu (JATS[2018]212).

* Corresponding author; e-mail: chenxuan@njau.edu.cn 
(Nagy et al. 2010). The expression patterns of $P R X s$ were different under normal growth and drought treatment in poplar leaves (Ren et al. 2014). But, there are no relevant research reports about the expression patterns of class III $P R X S$ in tea plants.

Abscisic acid (ABA), is a plant hormone which is known to be involved in plant responses to abiotic stresses such as high or low temperature, drought, and salinity (Nambara and MarionPoll 2005). It can induce stomatal closure and so reduce transpiration (Kazuo and Kazuko 2007). Methyl jasmonate (MeJA), is a growth regulator in plants, and it can also be involved in response to various biotic and abiotic stresses. It can conduct stress signals and initiate expression of stress-tolerant genes to protect the plants. The application of exogenous MeJA could enhance the drought resistance of plants by regulating expression of genes relating to antioxidant system. Few researchers has determined that interaction of jasmonic acid (JA) signal to ABA signal (Aleman et al. 2016).

In this paper, the expression of $C s P R X s$ and POD activity were examined in different tissues during drought stress. The aim was to determine potential functions of CsPRXs in tea plants and whether exogenous ABA and MeJA can increase tea tolerance to drought stress.

\section{Materials and methods}

Plant growth and treatment: The 2-year-old tea [Camellia sinensis (L.) Kuntze] cvs. Longjing 43 (LJ43) and Baiye 1 were purchased from Yarun Tea Company, Nanjing, Jiangsu Province, China and they were grown in an experimental field of Nanjing Agricultural University (Nanjing, China; latitude: $32.04^{\circ} \mathrm{N}$, longitude: $118.78^{\circ} \mathrm{E}$ ). The tea plants were cultured in a nutrient solution for $15 \mathrm{~d}$. After that, the young leaves, stems, and roots of both tea cultivars were collected and stored at $-80{ }^{\circ} \mathrm{C}$ to determine the expressions of CsPRXs in different tissues. To study the impact of hormones on tea plants under the drought conditions, 175 tea plants were divided into different groups. The control (CK) group was sprayed with distilled water, the ABA and MeJa groups were sprayed with $250 \mathrm{~cm}^{3}$ of $50 \mathrm{mg} \mathrm{dm}^{-3} \mathrm{ABA}$ or $0.2 \mathrm{mM} \mathrm{MeJA}$ at 8:00 every day for $3 \mathrm{~d}$, respectively. On the fourth day, $15 \%(\mathrm{~m} / \mathrm{v})$ polyethylene glycol 6000 (PEG 6000) was added to the solution of all groups. Then the first and second leaves were picked at $0,4,8,12,24,48$, and $72 \mathrm{~h}$, respectively (Ramírez et al. 2009). All the samples were put in liquid nitrogen and stored at $-80{ }^{\circ} \mathrm{C}$ for RNA extraction and the assessment of POD activity. Each sample contained three biological replicates.

Extraction of RNA and cDNA reverse transcription: Total RNA was extracted from $1 \mathrm{~g}$ of samples using the EASYspin Plus plant RNA rapid extraction kit (Takara, Tokyo, Japan). For reverse transcription of RNA into cDNA PrimeScript ${ }^{\mathrm{TM}}$ RT reagent kit with gDNA eraser (Takara) were used.

Cloning CsPRXs family: The target sequences were selected from the tea plant genome (www. plantkingdomgdb.com/tea tree/) and the primers of the open reading frames (ORFs) were designed (Table 1 Suppl.). Primers were synthesized at Anhui General Biosystems Company (Chuzhou, Anhui Province, China). The CsPRXs were amplified by PCR and detected by $1 \%(\mathrm{~m} / \mathrm{v})$ gel electrophoresis. The purified product was recovered and ligated with $p E A S Y-T 1$ Simple for sequencing.

The cloned CsPRXs were aligned using the sequences of other species from $N C B I$ (http://www.ncbi.nlm.nih.gov/) site by DANMAN software. The similarity of CsPRXs was obtained by comparison at www.genome.jp/tools-bin/ clustalw website. Proteins translated from ORFs were used to predict physical and chemical parameters: including calculation of molecular mass (Mr) and theoretical isoelectric point (pI) by ProtParam (http://web.expasy.org/ protparam/). Subcellular localization of target proteins was predicted using ProtComp v. 9.0 (http://psort.hgc.jp/form. html) online software. TMHMM Server v. 2.0 (http://www. cbs.dtu.dk/services/TMHMM-2.0/) was used to predict the number of membrane helices (TMHs) in transport proteins. The phylogenetic tree was constructed with the class III PRXs of Camellia sinensis, Arabidopsis thaliana, Vitis vinifera, and Pyurs bretschneideri based on MEGA 5.0 software (Tamura et al. 2011). Domain analysis of gene families and calculation of amino acid residue frequencies were performed using the Motif Elicitation (MEME) site (http://meme-suite.org/tools/meme). CsPRXs proteinprotein interaction networks were constructed based on the database of Arabidopsis thaliana by STRING (v. 10.5) (http://string-db.org/).

Expression of $\boldsymbol{C s P R X s}$ in two tea cultivars: The RTqPCR analysis was carried out by Bio-Rad (Hercules, USA) CFX96 Touch TM Deep Well real-time PCR detection system using TB Green ${ }^{T M}$ Premix Ex Taq ${ }^{\mathrm{TM}}$ (Takara) based on the manufacturer's protocol. The relative expressions of $C s P R X s$ were estimated by the $2^{-\Delta \Delta \mathrm{CT}}$ method (Livak and Schmittgen 2001). Primers for reverse transcription quantitative PCR were synthesized at Anhui General Biosystems Company (Table 2 Suppl.).

Assay of POD activity: For the POD activity analysis, $0.1 \mathrm{~g}$ sample was ground using liquid nitrogen in $0.9 \mathrm{~cm}^{3}$ of $0.9 \%(\mathrm{~m} / \mathrm{v}) \mathrm{NaCl}$, then extract was centrifuged at $12000 \mathrm{~g}$ for $10 \mathrm{~min}$. The supernatant was used to determine the POD activity by the imaging reader (Cytation3, BioTek, Winooski, VT, USA) in Nanjing Jiancheng Bioengineering Institute, China (Li et al. 2018). One unit (U) was defined as the change of absorbance at $470 \mathrm{~nm}$ of 0.01 per min.

Statistics: Experiments were carried out with three replicates. The statistical analyses were performed using Excel and the HemI1.0 software (Deng et al. 2014).

\section{Results}

A total of 17 candidate gene sequences of class III PRXS 
LI et al.

Table 1. Physical and chemical properties of $P R X s$ and predicted proteins in tea (Camellia sinensis) cv. LJ43 (ORF - open reading frame, pI - theoretical isoelectric point, $\mathrm{Mr}$ - relative molecular mass, ER - endoplasmic reticulum, TMHs - transmembrane helix).

\begin{tabular}{lclllll}
\hline Gene name & ORF length [bp] & Protein length [aa] & $\mathrm{Mr}[\mathrm{kDa}]$ & $\mathrm{pI}$ & Localization & Number of TMHs \\
\hline CsPRX3 & 981 & 326 & 35.28 & 8.96 & extracellular & 1 \\
CsPRX4 & 969 & 322 & 35.08 & 9.74 & mitochondrial & 1 \\
CsPRX12 & 1080 & 359 & 39.21 & 5.34 & ER & 1 \\
CsPRX16 & 990 & 329 & 36.11 & 8.11 & extracellular & 1 \\
CsPRX27 & 981 & 326 & 35.48 & 9.55 & extracellular & 0 \\
CsPRX42 & 1002 & 333 & 37.59 & 7.63 & extracellular & 0 \\
CsPRX43 & 972 & 323 & 34.64 & 4.62 & ER & 0 \\
CsPRX47 & 960 & 319 & 35.01 & 5.98 & ER & 1 \\
CsPRX55 & 975 & 324 & 34.69 & 4.68 & cytoplasmic & 0 \\
CsPRX72 & 984 & 327 & 35.84 & 8.99 & extracellular & 1 \\
CsPRX73 & 984 & 327 & 35.43 & 9.17 & nuclear & 0 \\
\hline
\end{tabular}

family were screened based on the tea plant genome (www.plantkingdomgdb.com/teatree/) and the NCBI database. After PCR cloning, ligation, transformation, and sequencing, 11 CsPRXs were obtained from leaves and uploaded to $N C B I$ (Genebank:2188835). The 11 sequences belonged to the $P R X$ family, and the amino acids at positions 25 to 320 had typical domains (Fig. 1 Suppl.). When comparing the amino acid sequences of the $11 \mathrm{CsPRXs}$, the similarity between CsPRX55 and CsPRX73 was the highest, and reached at $61.11 \%$, while between CsPRX42 and CsPRX43 was the lowest (25.69\%; Fig. 2 Suppl.). Physical and chemical properties are summarized in Table 1. The nucleotide sequences had open reading frames of 960 to $1080 \mathrm{bp}$, and the translated proteins had 319 to 359 amino acids, 34.64 to $39.21 \mathrm{kDa}$, and $\mathrm{pI}$ of 4.62 to 9.74 .. Five of the 11 CsPRXs were predicted to be in extracellular region. Among them, CsPRX3, CsPRX16, and CsPRX72 had transmembrane helixes (TMHs), while CsPRX27, CsPRX42 not. In addition, class III PRXs were present in different parts of the cell. CsPRX12, CsPRX43, and CsPRX47 were located in the endoplasmic reticulum. CsPRX4, CsPRX55, and CsPRX73 were located in mitochondria, cytoplasm, and nucleus, respectively. CsPRX4, CsPRX12, CsPRX43 had the TMHs, while CsPRX47, CsPRX55 and CsPRX73 not.

To investigate the genetic distance and phylogenetic relationships of class III PRXs among Camellia sinensis, Arabidopsis thaliana, Vitis vinifera, and Pyrus bretschneideri, the neighbor-joining method was used to construct phylogenetic tree with MEGA 5.0 software. According to Michael Tognolli's grouping (Dunand et al. 2002) of class III PRXs in Arabidopsis thaliana, they were divided into 5 sub-groups (Fig. 1). The genetic relationship of CsPRXs was closer to that of VitPRXs and PbPRXs. The number of CsPRX distributed in Grl subgroup was the highest, accounting for $45.45 \%$. While based on genetic distances, CsPRX4 and CsPRX27 showed a close relationship. CsPRX3, CsPRX47, and CsPRX55 were clustered in Gr2. CsPRX43, CsPRX12, and CsPRX42 were individually divided into Gr3, Gr4, and Gr5. To further understand the functional domain of CsPRXs, the MEME software was used. Eight of the 11 CsPRXs (CsPRX3, CsPRX12, CsPRX16, CsPRX27, CsPRX47, CsPRX55, and CsPRX72) contained 7 motifs, while CsPRX42, CsPRX43, and CsPRX72 had 6 motifs, and CsPRX4 had only 5 motifs (Fig. 2).

Using Arabidopsis thaliana as control, the the proteinprotein interactions were predicted on the STRING (v. 10.5) website. The PRXs corresponding relationship was determined according to the identity of sequences between Arabidopsis thaliana and tea plant. RCI3 (rare cold inducible gene 3) and AT5G66390 (peroxidase superfamily protein) had high similarity with CsPRX3 and CsPRX72, respectively. RCI3 and AT5G66390 were co-expressed in Arabidopsis thaliana (Fig. 3 Suppl.). Therefore, it was speculated that CsPRX3 and CsPRX72 were also co-expressed. In addition, AT4G37530 (peroxidase superfamily protein) and CAD5 (cinnamyl alcohol dehydrogenase 5), RCI3, and UGT72E3 (UDPglycosyltransferase superfamily protein), PRXR1 and UGT72E3 were co-expressed in Arabidopsis thaliana. However, there were no sequences in tea plant that were high similarity to CAD5 and UGT72E3.

In order to further explore the function of $C s P R X s$, qRT-RCR was used to determine the relative expression of $C s P R X s$ in the roots, stems, and leaves of the two tea cultivars. The 'LJ43' had a normal dark green colour, but Baiye 1 was a temperature-sensitive albino cultivar, whose leaves were light green (Fig. 4 Suppl.). The heat map shows the different expression patterns of the 11 CsPRXs in the two cultivars (Fig. 3). In 'LJ43', the relative expression of CsPRX55 was higher in roots than in stem and leaves. While, CsPRX42 and CsPRX72 were more expressed in stem than in leaves and roots. But in 'Baiye 1', the relative expressions of $C s P R X 12, C s P R X 55$, and $C s P R X 73$ were more abundant in the roots than in stem and leaves, while the expressions of $C s P R X 4, C s P R X 47$, and $C s P R X 72$ were the highest in the stem. The CsPRX3, CsPRX16, CsPRX27, and $C s P R X 43$ showed relatively low expressions in all three tissues of both cultivars, but relatively higher in leaves.

In order to compare the response of $C s P R X s$ to drought, 


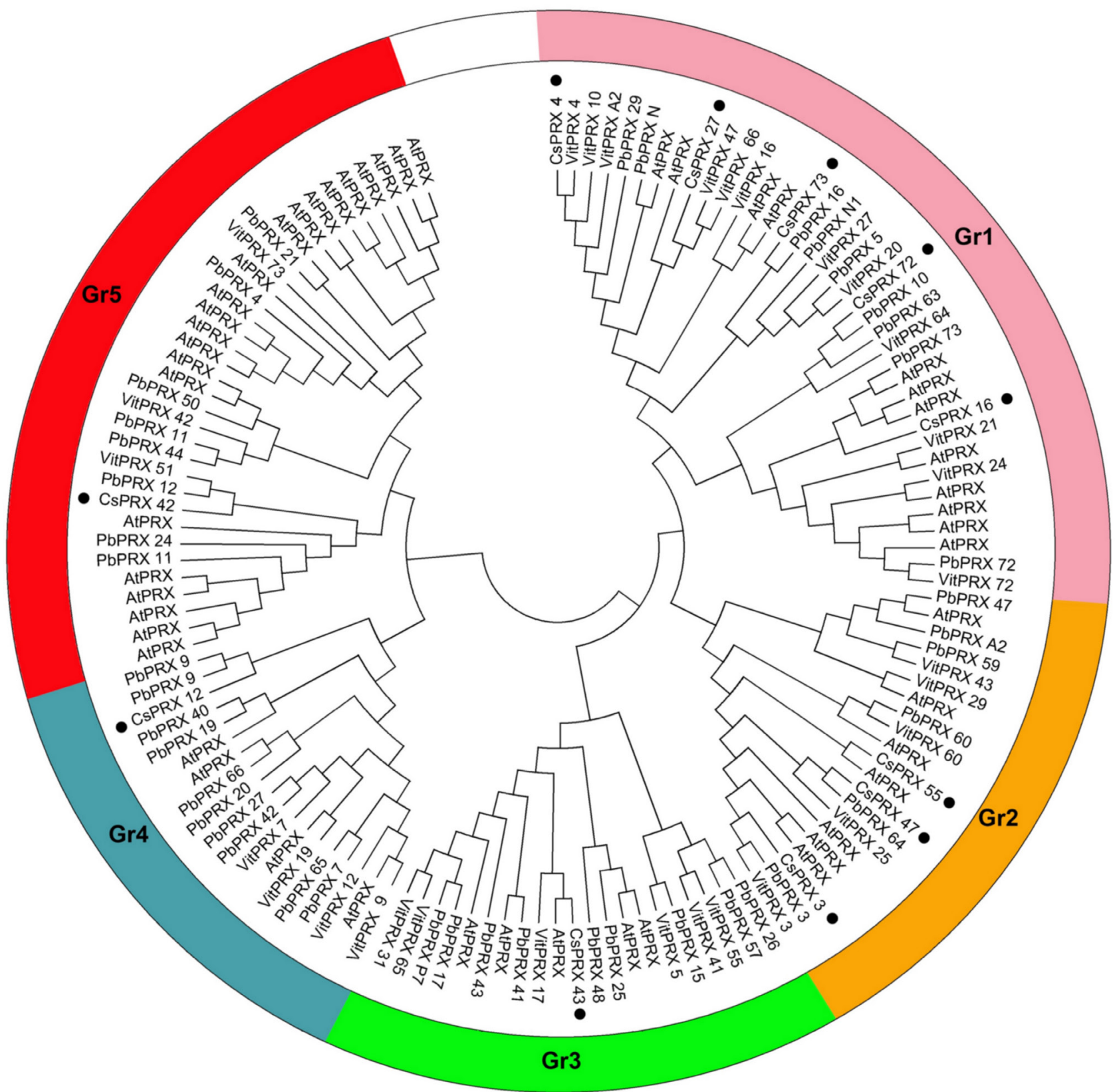

Fig. 1. A phylogenetic tree of PRXs from Camellia sinensis, Arabidopsis thaliana, Vitis vinifera, and Pyrus bretschneideri.

the expressions of CsPRXs was determined by RT-qPCR using the tea plants treated with PEG and pretreated with ABA or MeJa. The response of CsPRXs to ABA and MeJa was more sensitive under drought than in the control plants. Among them, CsPRX3, CsPRX27, and CsPRX43 were most important in response to ABA and MeJA treatment under drought stresses (Fig. 4). In the CK group, CsPRX3,CsPRX4,CsPRX12,CsPRX16,CsPRX27, and CsPRX47 showed significant responses to phytohormones in 'Baiye 1' compared with only CsPRX4 and CsPRX16 in 'LJ43'. In both cultivars, there were 3 CsPRXs (CsPRX3, CsPRX27, and CsPRX43) and 7 CsPRXs (CsPRX3,
CsPRX4, CsPRX12, CsPRX16, CsPRX27, CsPRX43, and $C S P R X 47)$ up-regulated after pretreatment with ABA and MeJA, respectively. Most of the CsPRXs reached the highest expressions at $4-12 \mathrm{~h}$ after treatment in 'LJ43', while the highest expression was observed between $12-24 \mathrm{~h}$ in 'Baiye 1'. In addition, CsPRX47 was expressed differently in the two tea cultivars (no changes in 'LJ43', but increase in 'Baiye 1').

The phenotype of the tea plant changed under drought stress. The leaf margin of the CK group showed more wilting at $72 \mathrm{~h}$ compared with $\mathrm{ABA}$ and MeJa treated groups (Fig. 5 Suppl.). The cv. 'Baiye 1' showed higher 


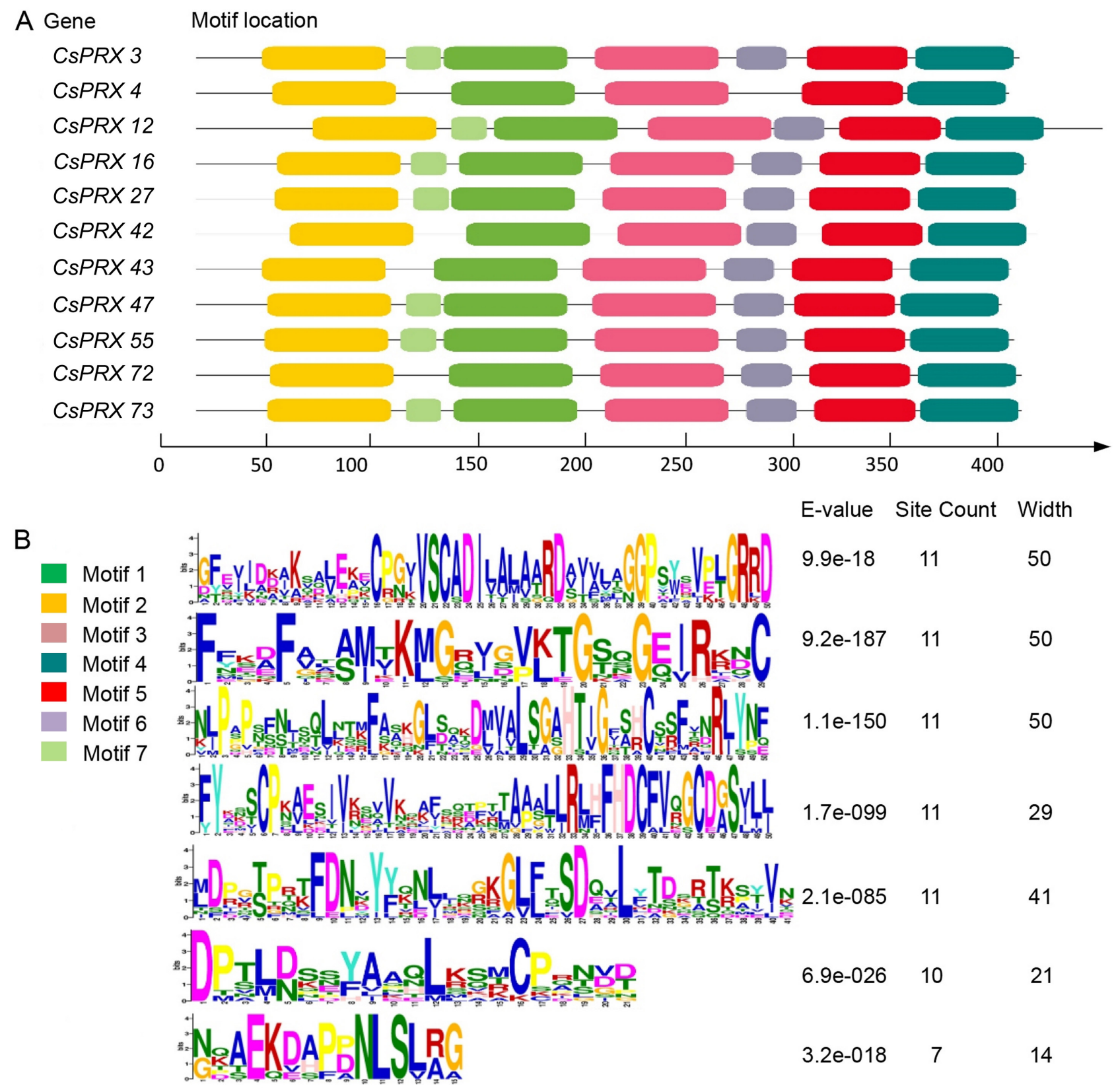

Fig. 2. Conserved sequences of PRXs from Camellia sinensis. The MEME program was used to search for patterns; pattern widths ranged from 15 to 50. $A$ - Residues in CsPRXs proteins were labelled with different colours. $B$ - The height of each letter represented the frequency occurrence of specific amino acids in each motif.

POD activity, so it may have a better cold resistance than 'LJ43' (Zhang et al 2017). But when suffered drought stress, the activity of POD of 'LJ43' was higher than that of 'Baiye 1' either in control plants or those pretreated with phytohormones (Fig. 5). The activity of POD in leaves increased significantly after ABA or MeJa pretreatments. In 'LJ43', the activity of POD was higher after ABA pretreatment than that in CK group from $4 \mathrm{~h}$ of drought stress; it reached the highest value at $24 \mathrm{~h}$; but it was reduced significantly at $72 \mathrm{~h}$. While under the MD treatment, the activity of the POD was always higher than that of CK group and it reached the highest level at $12 \mathrm{~h}$ of drought. In 'Baiye 1', the activity of POD after ABA and MeJA pretreatments was always higher than that of CK groups. The peak activity was at $24 \mathrm{~h}$ of drought stress when pre-treated by MeJA, but the change had no obvious rule after ABA pretreatment.

\section{Discussion}

The amino acid sequences of PRXs showed a relatively conservative evolution over a long period of evolution. Due to the choice of environment, the sequences of the 


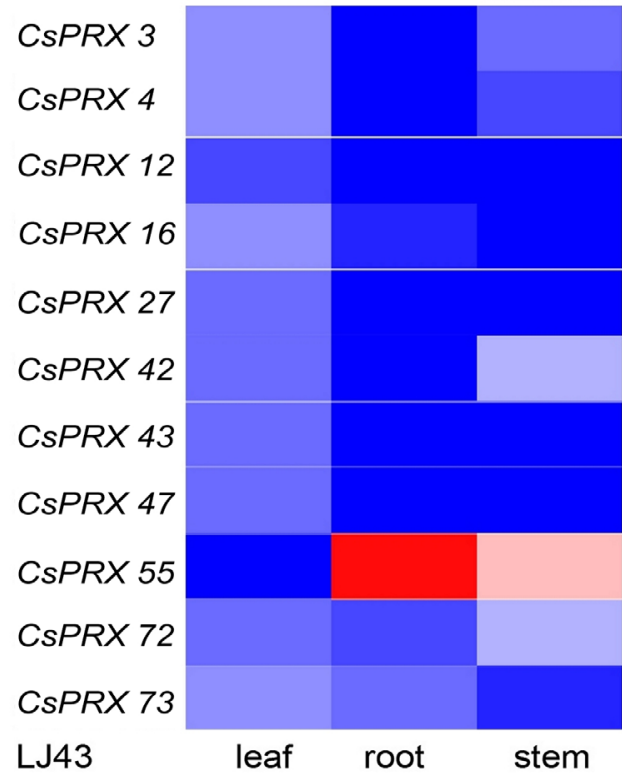

Fig. 3. The expression pattern of $C s P R X s$ in different tissues.

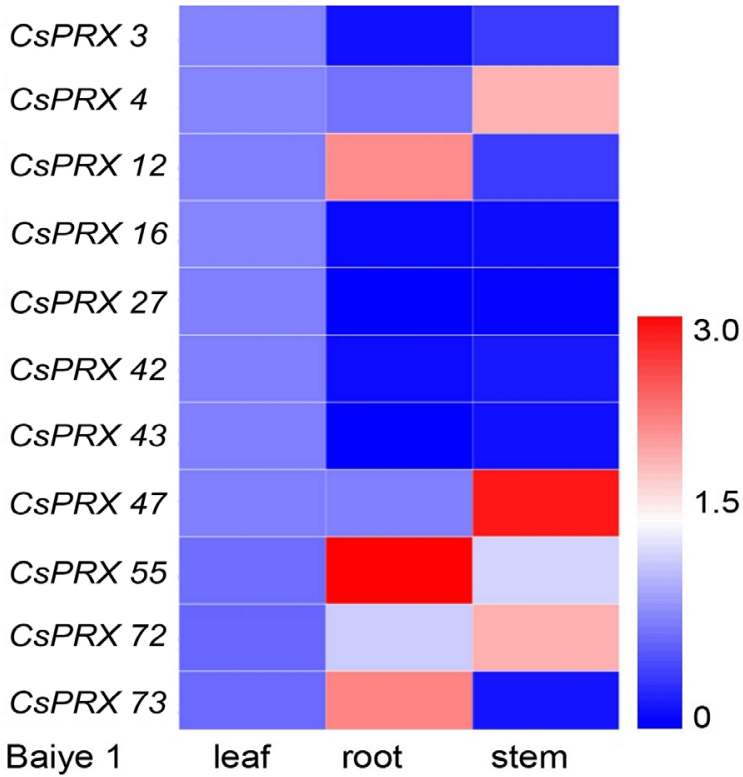

multi-gene family exhibited diversity (Wu et al. 2009). The class III PRX family were involved in the regulation of plant growth stages. The numbers of class III PRX family members were 73, 138, 93, 117, and 94, in Arabidopsis thaliana, Oryza sativa, Populus euphratica, Zea mays, and Pyrus bretschneideri, respectively (Michael et al. 2002, Passardi et al. 2004, Wang et al. 2015b, Cao et al. 2016). The publication of the tea plant genome facilitated the study of CsPRXs (Xia et al. 2017). In this study, 11 CsPRXs were cloned from the leaves and extensively discussed by analyzing the classification as well as structure and function. The conserved domain included the heme repair groups and the sites of binding substrate and $\mathrm{Ca}^{2+}$ in all CsPRXs. The identity of class III CsPRXs was between 25.69 and $61.11 \%$ (Fig. 2 Suppl.). However, the identity of AtPRXs was between 35 and $94 \%$ in the seven mature of Arabidopsis thaliana plants (Kjærsgård et al. 1997). Phylogenetic analysis had been carried out in order to understand the relationship between CsPRXs and other plant PRXs. The CsPRXs were divided into 5 sub-groups (Gr1-5). We found only one CsPRX in each of the Gr3, Gr4, and Gr5 sub-groups. But this did not mean that there was only one CsPRX in these three sub-groups. Referring to the number of PRX family in other plants, the number of CsPRX may be more in each sub-group. However, according to the PRX grouping of Rosaceae plants, they were divided into 21 sub-groups. And there were no PRX of Pyrus bretschneideri and Prunus mume, respectively, they were divided into 21 sub-groups (Cao et al. 2016). As can be seen from Fig. 1 and Fig. 2, the phylogenetic relationship of CsPRXs was consistent with the results of conserved motifs. This demonstrated the reliability of the experimental results.

There were large differences in gene expressions among different cultivars of tea plants (Wang et al. 2014). Due to their different genetic backgrounds, there were huge genetic variations in the germplasm resource of tea plants (Wachira et al. 2001). In this study, the relative expressions of CsPRXs were not high in various tissues of 'LJ43' (except CsPRX55). However, CsPRX4, CsPRX12, CsPRX47, CsPRX55, CsPRX72, and CsPRX73 were strongly expressed in roots or stems in 'Baiye 1' (Fig. 3). Sixteen of $73 A t P R X S$ were strongly expressed in 2-4 tissues (Valério et al. 2004). This suggested that these genes may play an important role in different tissues as the expression profile of genes is associated with their function (Burbridge et al. 2014, Wang et al. 2015a). According to the Gr1 sub-group of phylogenetic tree constructed in this paper, CsPRX4, CsPRX16, CsPRX27, CsPRX73 had the highest expression in leaves, but the highest expression of $C s P R X 72$ was in 'LJ43' stems (Fig. 3). The expression pattern of CsPRXs was not always based on predicted results. The expression and function of genes were not all the same in the same sub-group, and even the expression patterns of low sequence similarity genes may be similar. Therefore, the constructed evolutionary tree did not seem to accurately infer the functional role or position of amino acids (Kumari et al. 2008, Beltramo et al. 2012).

Many studies reported the correlations between ABA or MeJA sensitivity and the expression of drought-related genes. ABA and MeJA act as signaling molecules that mediated plant growth and response to stresses (Lee and Lin 1996). ABA was indispensable for plant adaptation to drought stress and ABA content increases in plant tissues, particularly in leaves, under water stress (Cutler et al. 2010). Hypersensitivity to ABA might be associated with the tolerance to drought stress due to stomatal closure and the expression of stress-related genes (Lim et al. 2014).

Class III PRXs were encoded by a large gene family in plants (Hiraga et al. 2001, Wang et al. 2016). The glycoproteins located in the vacuole and the class III PRXs in the cell wall could catalyze the disproportionation 

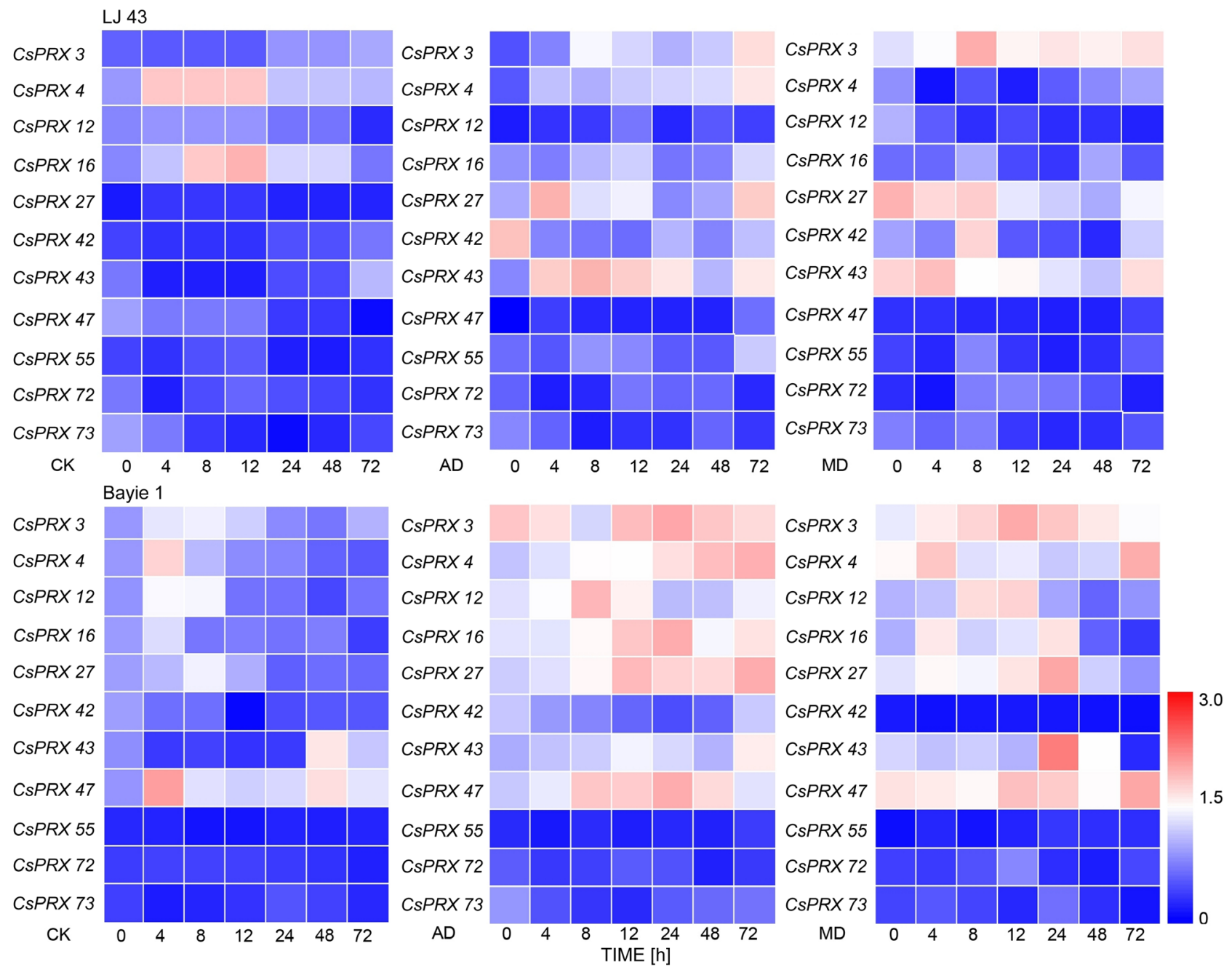

Fig. 4. Expression patterns of the CSPRXs in response to drought induced by $15 \%$ PEG 6000. Plants were pretreated with water or phytohormones. CK - water + drought; ABA - $50 \mathrm{mg} \cdot \mathrm{dm}^{-3} \mathrm{ABA}+$ drought; MeJa - $0.2 \mathrm{mM}$ MeJA + drought. The CsACT was used as a housekeeping gene and the mean value of three replicates was used to generate a heat map using the HemI1.0 software. The bluewhite-red scheme was labeled on the right side of the heat map. Red and blue represent relatively higher and lower expression levels, respectively, than those in the $0 \mathrm{~h}$ drought group.

of superoxide radical ions into $\mathrm{H}_{2} \mathrm{O}_{2}$ as part of the plant defence (Almagro et al. 2008). PpPRXs also showed different expression patterns under $\mathrm{H}_{2} \mathrm{O}_{2}, \mathrm{SA}$, drought, and salt treatments in Populus (Ren et al. 2014). In this study, the $11 C s P R X s$ had different expression patterns under ABA and MeJa treatments (Fig. 4). Many PRXs are identified to help plants adapt to environmental stresses (Wang et al. 2015). Compared to the control group, the application of ABA or MeJA increased the expression of the $C s P R X s$ in tea plants, which could help them grow well under drought stress (Fig. 4). In addition, the expression level of the same gene also varied between LJ 43 and BY 1, e.g., the CsPRX47 was differently expressed in these two cultivar. Also, the expression of $C s P R X 4$ was down-regulated after ABA and MeJa treatmnt only in 'LJ43'. This indicates that the albino cultivar Baiye 1 was changed not only in chloroplast development, but also in gene transcription regulation and the antioxidant function. Using Arabidopsis thaliana as control, the protein-network map of CsPRXs was predicted, which provided a basis for studying whether CsPRXs were coexpressed in tea plants. Based on the similarity of amino acid sequences of of PRXs between Camelia sinensis and Arabidopsis thaliana, we speculated that CsPRX3 and CsPRX72 could co-express (Fig. 3 Suppl.), but they did not co-express at any tissue and at any treatment. This may require further reaserch using other methods of clustering. Drought stress causes rapid damage of cell membranes due to lipid peroxidation by drought-induced reactive oxygen species (DaCosta and Huang 2007). POD is one of the important enzymes that effectively eliminated $\mathrm{H}_{2} \mathrm{O}_{2}$, e.g in rubber trees (Wang 2014). ABA can regulate the content of $\mathrm{H}_{2} \mathrm{O}_{2}$ by controlling POD activity. The POD activity came from all the expressoions of $C s P R X$. When a plant is subjected to abiotic stress, the genes usually began to respond within a few hours, but it took a day or even a few days to change the protein composition or even the phenotype. We also observed changes in expression of 


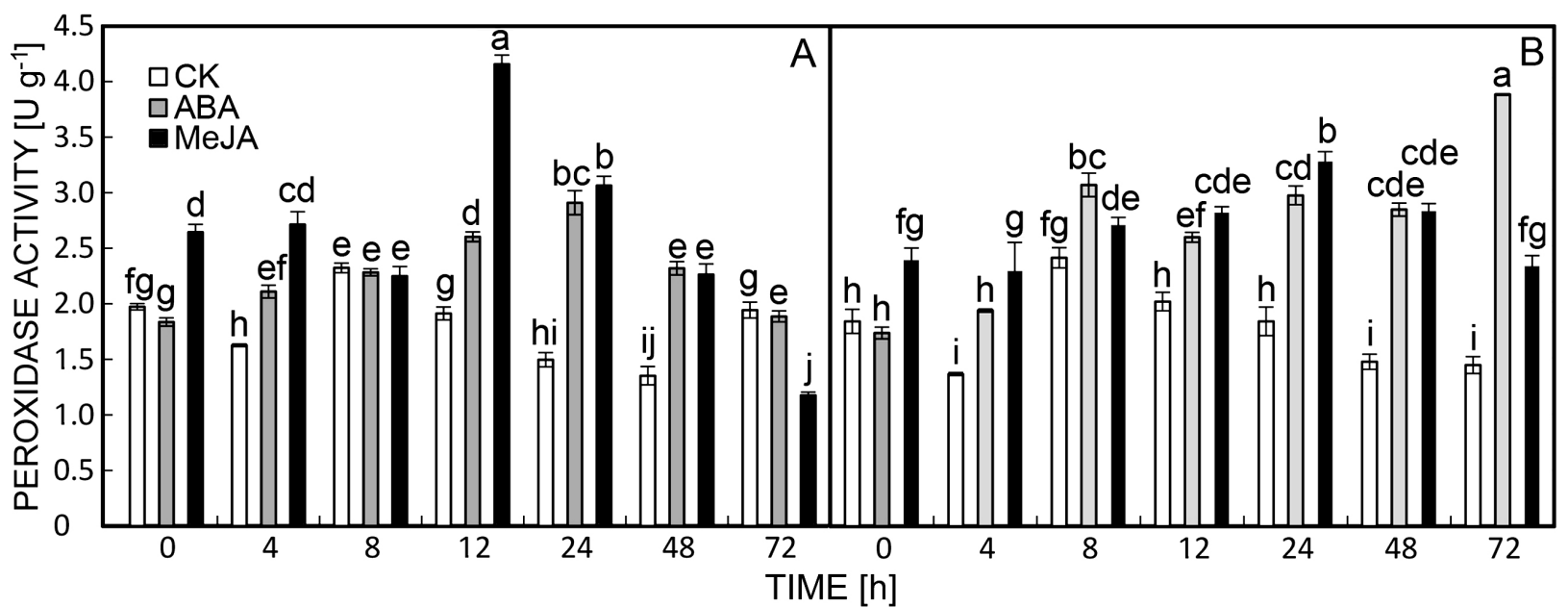

Fig. 5. Effects of abscisic acid (ABA) and methyl jasmonate (MeJA) on peroxidase activity of tea plants during the drought period. CK - water + drought treatment; ABA - ABA + drought; MeJa - MeJA + drought. The data were analyzed using GraphPad Prism 5. Means $\pm \mathrm{SDs}, n=3$. Statistically significant differences at $P<0.05$ are indicated with different letters.

CsPRXs within the first $8 \mathrm{~h}$ of drought stress (Fig. 4), there was almost no difference in the POD activity during this period. The time necessary for increase in POD activity lasted longer in 'Baiye 1' than in 'LJ43'. Peroxidase is a stress-responsive substance in plant hormone signaling pathways. These pathways, regulated by ABA, MeJa, as well as reactive oxygen species signaling, play key roles in crosstalk between biotic and abiotic stress signaling. (Fujita et al. 2006). In 'LJ43', the time to the peak of POD activity in ABA treatment was $12 \mathrm{~h}$ later than in MeJa treatment, whereas in 'Baiye 1', it was $48 \mathrm{~h}$ later in ABA treatment compared with MeJa treatment (Fig. 5). The time necessary for increasing POD activity was longer in leaves pre-treated with ABA than that with MeJA. This may be due to MeJA that was more effective against drought stress. (Hassanein et al. 2009).

In conclusion, the study analyzed the bioinformatics about the 11 cloned CsPRXs. The tissue specificity in Baiye 1 was more obvious than in 'LJ43' as revealed by the gene expressions in tissues (roots, stems, and leaves). Compared with the different expression patterns of CsPRXs in roots and stems, the leaves were consistent in the two cultivars. In addition, compared with 'LJ43', the increase in POD activity in 'Baiye 1' was delayed after treatments with ABA and MeJA. This suggests that the response of 'Baiye 1' to plant exogenous growth regulators may be hindered. What is more, CsPRX3, CsPRX27, and CsPRX43 were the most important CsPRXs involved in the response to drought stress after different hormone pretreatments in tea plant. This provided a theoretical basis for preventing drought and improving the yield and quality of tea.

\section{References}

Aleman, F., Yazaki, J., Lee, M., Takahashi, Y., Kim, A.Y., Li, Z.X., Kinoshita, T., Ecker, J.R., Schroeder, J.I.: An ABAincreased interaction of the PYL6 ABA receptor with MYC2 transcription factor: a putative link of $\mathrm{ABA}$ and JA signaling. - Sci. Rep. 6: 28941, 2016.

Almagro, L., Gómez Ros, L., Belchi-Navarro, S., Bru, R., Ros Barceló, A., Pedreno, M.: Class III peroxidases in plant defence reactions. - J. exp. Bot. 60: 377-390, 2008.

Bartels, D., Sunkar, R.: Drought and salt tolerance in plants. Crit. Rev. Plant Sci. 24: 23-58, 2005.

Beltramo, C., Marinoni, D.T., Perrone, I., Botta, R.: Isolation of a gene encoding for a class III peroxidase in female flower of Corylus avellana L. - Mol. Biol. Rep. 39: 4997-5008, 2012.

Burbridge, E., Rasmussen, S.K., Bernier, F., Kristensen, B.K., McCabe, P.F., Dix, P.J.: Altered activity of peroxidase and oxalate oxidase influences lignification in transgenic tobacco. - Open Plant Sci. J. 8: 1-8, 2014.

Cao, Y.P., Han, Y.H., Meng, D.D., Li, D.H., Jin, Q., Lin, Y., Cai, Y.P.: Structural, evolutionary, and functional analysis of the class III peroxidase gene family in Chinese pear (Pyrus bretschneideri). - Front. Plant Sci. 7: 1874, 2016.

Cosio, C., Dunand, C.: Specific functions of individual class III peroxidase genes. - J. exp. Bot. 60: 391-408, 2008.

Cutler, S.R., Rodriguez, P.L., Finkelstein, R.R., Abrams, S.R.: Abscisic acid: emergence of a core signaling network. - Annu. Rev. Plant Biol. 61: 651-679, 2010.

DaCosta, M., Huang, B.R.: Changes in antioxidant enzyme activities and lipid peroxidation for bentgrass species in response to drought stress. - J. amer. Soc. hort. Sci. 132: 319326, 2007.

Deng, W., Wang, Y., Liu, Z., Cheng, H., Xue, Y.: HemI: a toolkit for illustrating heatmaps. - PloS ONE 9: e111988, 2014.

Dunand, C., Tognolli, M., Overney, S., Tobel, L., Meyer M, Simon, P., Penel,C.: Identification and characterisation of $\mathrm{Ca}^{2+}$-pectate binding peroxidases in Arabidopsis thaliana. J. Plant Physiol. 159: 1165-1171, 2002.

Fujita, M., Fujita, Y., Noutoshi, Y., Takahashi, F., Narusaka, Y., Yamaguchi-Shinozaki, K., Shinozaki, K.: Crosstalk between abiotic and biotic stress responses: a current view from the points of convergence in the stress signaling networks. - Curr. Opin. Plant Biol. 9: 436-42, 2006.

Hassanein, R.A., Hassanein, A.A., El-Din, A.B., Salama, M., Hashem, H.A.: Role of jasmonic acid and abscisic acid treatments in alleviating the adverse effects of drought stress and regulating trypsin inhibitor production in soybean plant. - 
Aust. J. basic. appl. Sci. 3: 904-919, 2009.

Hiraga, S., Sasaki, K., Ito, H., Ohashi, Y., Matsui, H.: A large family of class III plant peroxidases.- Plant Cell Physiol. 42: 462-468, 2001

Kazuo, S., Kazuko, Y.S.: Gene networks involved in drought stress response and tolerance. - J. exp. Bot. 58: 221-227, 2007.

Kjærsgård, I.V., Jespersen, H.M., Rasmussen, S.K., Welinder, K.G.: Sequence and RT-PCR expression analysis of two peroxidases from Arabidopsis thaliana belonging to a novel evolutionary branch of plant peroxidases. - Plant mol. Biol. 33: 699-708, 1997.

Kumari, M., Taylor, G.J., Deyholos, M.K.: Transcriptomic responses to aluminum stress in roots of Arabidopsis thaliana. - Mol. Genet. Genomics 279: 339-357, 2008.

Kunieda, T., Shimada, T., Kondo, M., Nishimura, M., Nishitani, K., Hara Nishimura, I.: Spatio-temporal secretion of peroxidase 36 is required for seed coat mucilage extrusion in Arabidopsis. - Plant Cel. 25: 1355-1367, 2013.

Lee, T.M., Lin, T.H.: Peroxidase activity in ethylene-, ABA-, or MeJA-treated rice (Oryza sativa L.) roots. - Bot. Bull. Acad. sin. 37: 201-207, 1996.

Li, J., Arkorful, E., Cheng, S., Zhou, Q., Li, H., Chen X, C., Sun, K., Li, X.: Alleviation of cold damage by exogenous application of melatonin in vegetatively propagated tea plant (Camellia sinensis (L.) O. Kuntze). - Sci. Hort. 238: 356-362, 2018.

Lim, S., Baek, W., Lee, S.C.: Identification and functional roles of CaDIN1 in abscisic acid signaling and drought sensitivity. - Plant mol. Biol. 86: 513-525, 2014.

Livak K.J, Schmittgen T.D.: Analysis of relative gene expression data using real-time quantitative PCR and the $2^{-\Delta \Delta C \mathrm{CT}}$ method. - Methods 25: 402-408, 2001.

McInnis, S.M., Costa, L.M., Gutiérrez Marcos, J.F., Henderson, C.A., Hiscock, S.J.: Isolation and characterization of a polymorphic stigma-specific class III peroxidase gene from Senecio squalidus L. (Asteraceae). - Plant mol. Biol. 57: 659$677,2005$.

Michael, T., Claude, P., Hubert, G., Patrice, S.: Analysis and expression of the class III peroxidase large gene family in Arabidopsis thaliana. - Gene 288: 129-138, 2002.

Mirzaee, M., Moieni, A., Ghanati, F.: Effects of drought stress on the lipid peroxidation and antioxidant enzyme activities in two canola (Brassica napus L.) cultivars. - J. agr. Sci. Technol. 15: 593-602, 2013.

Nagy, N.E., Fossdal, C.G., Dalen, L.S., Lönneborg, A., Heldal, I., Johnsen.: Effects of Rhizoctonia infection and drought on peroxidase and chitinase activity in Norway spruce (Picea abies). - Physiol. Plant. 120: 465-473, 2010.

Nambara, E., MarionPoll, A.: Abscisic acid biosynthesis and catabolism. - Ann. Rev. Plant Biol. 56: 165-185, 2005.

Passardi, F., Longet, D., Penel, C., Dunand, C.: The class III peroxidase multigenic family in rice and its evolution in land plants. - Phytochemistry 65: 1879-1893, 2004.
Ramírez, V., Coego, A., López, A., Agorio, A., Flors, V, Vera, P.: Drought tolerance in Arabidopsis is controlled by the OCP3 disease resistance regulator. - Plant J. 58: 578-591, 2009.

Ren, L.L., Liu, Y.J., Liu, H.J., Qian, T.T., Qi, L.W., Wang, X.R., Zeng, Q.Y.: Subcellular relocalization and positive selection play key roles in the retention of duplicate genes of Populus class III peroxidase family. - Plant Cell 26: 2404-2419, 2014.

Sharma, A.D., Rakhra, G., Kaur, S., Kaur, H.: Expression of boiling-stable peroxidase (PRX) isoenzymes under combined effect of drought and heat in different tissues of Triticum aestivum. - Int. J. Agr. Technol. 9: 901-913, 2013.

Tamura, K., Peterson, D., Peterson, N., Stecher, G., Nei, M., Kumar, S.: MEGA5: molecular evolutionary genetics analysis using maximum likelihood, evolutionary distance, and maximum parsimony methods. - Mol. Biol. Evol. 28: 2731$2739,2011$.

Valério, L., De Meyer, M., Penel, C., Dunand, C.: Expression analysis of the Arabidopsis peroxidase multigenic family. Phytochemistry 65: 1331-1342, 2004.

Wachira, F., Tanaka, J., Takeda, Y.: Genetic variation and differentiation in tea (Camellia sinensis) germplasm revealed by RAPD and AFLP variation. - J. hort. Sci. Biotech. 76: 557563, 2001.

Wang, L.F.: Physiological, molecular responses to drought stress in rubber tree (Hevea brasiliensis Muell. Arg.). - Plant Physiol. Biochem. 83: 243-249, 2014.

Wang, L., Yue, C., Cao, H.L., Zhou, Y.H., Zeng, J.M., Yang, Y.J., Wang, X.C.: Biochemical and transcriptome analyses of a novel chlorophyll-deficient Chlorina tea plant cultivar. - BMC Plant Biol. 14: 352, 2014.

Wang, L.F., Wang, J.K., An, F., Xie, G.S.: Molecular cloning and characterization of a stress responsive peroxidase gene HbPRX42 from rubber tree. - Braz. J. Bot. 39: 475-483, 2016.

Wang, Y., Chen, J.W.: A comparative study on the SOD, POD, MDA and dissoluble protein of six provenances of Chinese pine (Pinus tabulaeformis Carr.) under water stress. - Ecol. environ. Sci. 20: 1449-1453, 2011.

Wang, Y., Wang, Q.Q., Zhao, Y., Han, G.M., Zhu, S.W.: Systematic analysis of maize class III peroxidase gene family reveals a conserved subfamily involved in abiotic stress response. - Gene 566: 95-108, 2015.

Wu, Y.R., Deng, Z.R., Lai, J.B., Zhang, Y.Y., Yang, C.P., Yin, B., Zhao, Q.Z., Zhang, L., Li, Y., Yang, C.W.: Dual function of Arabidopsis ATAF1 in abiotic and biotic stress responses. Cell Res. 19: 1279-1290, 2009.

Xia, E.H., Zhang, H.B., Sheng, J., Li, K., Zhang, Q.J., Kim, C., Zhang, Y., Liu, Y., Zhu, T., Li, W.: The tea tree genome provides insights into tea flavor and independent evolution of caffeine biosynthesis. - Mol. Plant. 10: 866-877, 2017.

Zhang, L., Li, X, Wei, J.P., Li, Z.X., Shen, C.,Yan, P., Zhang, L.P., Han, W.Y.. [Analysis of the cold resistance of Baiye 1.] - J. Tea Sci. 37: 71-77, 2017. [In Chinese] 